the artery, and was quite loose in the wound. The common iliac artery was healthy both above and below where it had been tied. The aneurismal sac had been completely emptied of its contents. Its coats were in a state of gangrene, which destroyed almost the whole of the external iliac artery. The gangrene had also extended to the surrounding tissues, and to the iliac and gluteal muscles, which were converted into a soft pulpous mass. There was a considerable quantity of grumous matter in the fossa of the ilium, similar to what had passed during life.

Although this case was unsuccessful, I have thought it necessary to publish it, as I only find on record those cases which have been successful-I believe about six in number.

Islington, Liverpool, August 1852.

\title{
ON SUPPURATION IN BONE.
}

By HENRY LEE, Esq., F.R.C.S., Surgeon to the Lock Hospital, Assistant Surgeon to King's College Hospital, etc.

(Continued from page 14, in Number for January 1852.)

\section{PURUIENT INFIITRATION OF BONE.}

WHEN suppuration takes place in bone, and the fluid is neither limited by a cyst, nor by condensed bony matter, it will escape into the surrounding cancellous structure. Any diseased secretions may in a similar way infiltrate a large portion of the interior of a bone, and may there produce much local and constitutional derangement. Unless relief be afforded by artificial means, the morbid matter has no means of escape, and can be removed only by being received into the circulation, or by the lengthened process of ulceration or necrosis.

The free communication which exists between different parts of the cancellous structure of a bone is illustrated by a very simple experiment. If a small hole be bored in the sides of an adult bone, and water be injected, it will without difficulty permeate the whole of its interior; or, if the experiment be performed upon the bone of a younger animal, (up to the time at which it attains its full development), the whole of the shaft will be injected, but none of the injection will penetrate to the epiphysis. In like manner, diseased fluids, when they escape into the cancellous structure of bone, beyond the limit prescribed by the adhesive inflammation, are confined only by the dense osseous parietes, or by the junction of the epiphysis with the shaft.

The accompanying wood-cut represents a section of a femur infiltrated with pus, after an injury which had exposed a large portion of the cancellous structure of its lower extremity.

The line of separation, formed by the junction of the epiphysis with the shaft of the bone, is of much importance with regard to operations involving the extremities of the long bones in young patients. Up to the age of seventeen, and perhaps later, the epiphysis may be separated from a long bone without injuring its structure. In such 


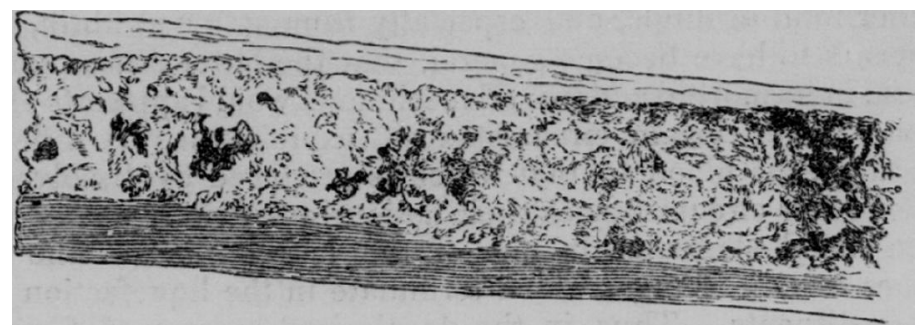

Fig. 5. Purulent Infiltration of Bone.

cases, however much the epiphyses of bones may be injured, (as, for instance, in the excision of joints), there is no probability that purulent or other infiltration will extend to the body of the bone, provided that has not itself been involved in the operation; or, on the other hand, if the shaft of the bone be the original seat of disease, that any morbid action will extend from it to the epiphysis. That diseased actions in bone are generally communicated by infiltration of the cancellous structure, and that such actions cease when they cannot thus be transmitted, is sometimes illustrated in the scrofulous affections of the bones of the fingers in children. The whole of the shaft of a bone may in such cases perish, leaving the articular extremities unaffected; recovery may then take place with a shortened finger, but without any interference with the natural motions of the joints.

The experiment of injecting the interior of a bone will not only show that a very free communication exists between the different parts of its cancellous structure, but also that the injected fluid may be made to pass very readily from the cancelli into the nutritious vessels of the bone, and thence into the general circulation. If the experiment be performed upon a bone which has just attained its full development, the injection will pass freely from the nutritious ressels of the shaft, or of the epiphysis, according to the situation in which the opening into the bone has been made.

It is easy therefore to understand that disease may readily be propagated, not only from one part of a bone to another, but also from the interior of a bone to different parts of the vascular system. This is doubtless the explanation of the very large proportion of cases, in which purulent infiltration of bone precedes secondary abscesses. In such instances, the course of the morbid action may be traced through the vessels of the bone into those of the general circulation. After amputation of a limb, terminating in phlebitis, for instance, it will sometimes happen that the divided extremities of the veins will show no signs of having participated in the disease, nor will any signs of inflammation be seen for several inches from the surface of the stump. But the first appearances of inflammation will appear in those veins, which are traversed by the blood derived from the nutritious vessels of the divided bone.

It is a point of much interest to trace the mode in which the fatal affection is in such cases transmitted. The following observations and experiments, while they are in some respects peculiarly applicable to secondary affections resulting from purulent infiltration of bone, are illustrative of similar diseases having their origin in other parts.

So much care has been taken of late years to distinguish purulent 
from other morbid fluids, and especially from softened fibrin, that the idea appears to have become general, that the latter differs essentially from them in its morbid actions. Experiments would appear to show that while pus, under certain circumstances, exercises a very different influence upon the blood to that which is produced by dissolved fibrin, yet under other circumstances their actions are strictly analogous.

When blood stagnates in one of the larger veins of the body, it undergoes certain changes which terminate in the liquefaction of some of its constituents. Thus, in the decolorised masses of fibrin found in the veins after death, it is not at all uncommon to find the central portions in a fluid state. This change is in exact accordance with Mr. Gulliver's experiments upon fibrin when removed from the body, but maintained at its natural temperature. It would appear, however, that this conversion takes place much more readily in certain conditions of the system than in others; and as, on the one hand, the fibro-albuminous portions of the blood may very readily assume the solid form, so on the other hand may these same constituents of the blood, after they have become solid, be reconverted into their fluid condition.

CAsE. A gentleman, of a gouty habit, had an attack of low, illdefined erysipelatous inflammation in the leg; at the same time the femoral vein could be felt as a thickened cord at the upper part of the thigh. An incision having been made in the leg, a small branch of the internal saphena vein was divided, and the blood flowed from it as it would have done from an artery, but without pulsation. This evidently depended upon the blood being prevented from following its natural course by the obstruction in the femoral vein. The blood which flowed from the wound was received on a sponge in some warm water. Almost immediately it separated into its different parts. The decolorised fibrin floated in firm shreds in the water, and adhered in the same condition to the sponge upon which it was received. Some of this fibrin was collected; and, having been separated as far as possible from any admixture of water, it was placed in a clean bottle and corked up. When collected, it was quite as firm as fibrin usually is, and it required some little pressure to force some of the portions through the neck of the bottle. No artificial heat was in this case applied, but on the following day, the whole of the contents of the bottle had become reconverted into their original fluid condition.

The readiness with which the fibro-albuminous portions of the blood may undergo the change from the fluid to the solid, and from the solid to the fluid form, is a point of considerable interest in tracing the mode in which purulent affections of bone may extend, first to the nutritious veins of the part, and subsequently to the general system. When fibrin, which has been allowed to become fluid by decomposition, is mixed with recently drawn blood, it will determine its rapid coagulation. In this respect, the action of dissolved fibrin is analogous to that of pus; but there is this important distinction between the two, that, whereas the coagulum formed by pus is particularly firm, that formed by decomposed fibrin is loose, and however long it is kept, it does not become more solid.

The experiments which I have before published, illustrating the peculiar action of pus upon'blood, have been repeated by several 
persons, and especially by Mr. Millington of Edinburgh. The observations of this gentleman led him to the conclusion, that, whereas pus added to living blood determined its rapid and firm coagulation, putrid fluids, on the contrary, either retarded or altogether prevented such an action. From experiments which I have myself made with putrid fluids, I believe this conclusion to be generally true. But the effect of putrid fibrin upon blood affords a marked and very peculiar exception. It is to be remembered, that the blood in the living being is peculiarly subject to the action of decomposed fibrin; and it is in perfect accordance with the usual economy of nature to find the sensibilities of a part peculiarly alive to those influences which are calculated to affect it injuriously. The blood forms no exception to this general rule. Its sensibilities for its self-preservation are as evident as those of other parts of the body, and are called into play in an especial manner in reference to those injurious influences to which it is peculiarly liable. It is seldom that the blood is subject to the direct action of putrid vegetable matters, or even to the putrid secretions from animal bodies. But it is in an especial manner open to the influence of portions of liquefied fibrin derived from blood, which for a time has stagnated in some part of the vascular system. The following experiments will show how readily the contact of such is felt by the living blood, and also the means adopted for the preservation of the general mass of the circulatory fluid from its deleterious influence.

Experiment. Some fibrin of the blood was obtained quite firm, free from any colouring matter, and was kept and allowed to decompose. At the expiration of three or four weeks, it had become fluid and of a dirty light brown colour; a small portion of this was now mixed with some recently drawn blood from a bullock which had just been knocked down. In two minutes from the commencement of the experiment the mixture had formed a uniform soft coagulum : a similar quantity of unmixed blood, taken at the same time, remained fluid several minutes afterwards.

This experiment was repeated with some of the same fibrin in a more advanced stage of decomposition. A half pint of blood was taken from a horse in perfect health, and to this was immediately added a quarter of an ounce of the putrid fluid. The whole coagulated loosely in a minute and a half. The coagulum was kept till the next day, but did not become firmer than it was after it had first coagulated. The effects obtained from the action of decomposed fibrin on living blood are thus shown to be different, both from those produced by ordinary putrid fluids on the one hand, and by pus on the other. The experiment appears to prove, that in case any putrid fibrin should find its way into a blood vessel, the same means would be in force to limit its influence, to seal the vessel, and to protect the system against its circulation, as would happen if some healthy pus had been similarly introduced. But the soft and loose coagulum in the one case, although as rapidly formed, affords much less protection to the constitution, than the firmer and adherent clot in the other. ${ }^{1}$

When the fluid fibrin has attained a very great degree of putridity,

It is important to notice that these observations apply only to fluid fibrin in a putrid state; whether the effects of fluid fibrin which had not undergone decomposition would be similar, I have not had an opportunity of ascertaining. 
the coagulum formed may not be of sufficient consistence to resist the circulating power of the blood. The morbid poison, when introduced into a vein, will under such circumstances pass without obstruction into the system. The effects produced will then offer a strong contrast to those which result from a similar introduction of pus into the circulation.

The following experiment illustrative of this point was performed for me by Mr. Mayer, veterinary surgeon. A young and perfectly healthy ass was secured, and the right jugular vein exposed. An ounce of fluid, derived from the spontaneous decomposition of portions of the fibrin used in the two former experiments, but in a much more advanced state of putrefaction, was mixed with an equal quantity of water, and injected by means of a syringe into the exposed vein. The vessel did not become "corded", and the circulation was not apparently impeded, as happens when a similar experiment is made with pus. In a few moments, the animal gave three or four groans expressive of much distress. The vital powers at the same time appeared to be suddenly prostrated. The animal was unable to rise, and soon fell into a state of syncope.

The faintness lasted for a few minutes. On getting up, the animal reeled and staggered about for some time, and then recovered sufficiently to walk to his stall. Some reaction now commenced, the breathing was disturbed, short, and quick; the pulse 120 in the minute, small and wiry. These symptoms continued for about an hour and a half, when the animal became very restless and uneasy, evincing internal pain by groaning and looking at his side. All food and water were refused, and death followed four hours after the operation.

On a post-mortem inspection sixteen hours after death, the jugular vein, into which the putrid fluid had been injected, was found in its natural condition, partially distended with fluid blood. It was pervious through its entire length, and contained no coagula. The lungs were studded with irregularly circumscribed, soft, black patches. When cut into, these discharged a black fluid, having the appearance of a mixture of blood and ink, and of a strong putrid smell. The heart had liquid blood in both its cavities. The crecum and colon and a portion of the small intestines were deeply congested, and of a dark livid colour. There was an effusion of many pints of serum of the peritoneal cavity; this, as well as the blood throughout the body, had a putrid and very unpleasant smell.

In this case, the putrid injection either prevented the coagulation of the blood in the body, both before and after death, or had the effect (in the event of any coagula having been formed) of determining their speedy reconversion into a fluid state. These results afford the strongest contrast to those produced by the introduction of pus into a vein. In the latter case, the immediate and firm coagulation of the blood presents a barrier against the admission of the contaminated blood into the circulation; or, if by any means this first means of localising the morbid matter should prove abortive, the tendency of the infected portion of the blood to coagulate is evinced in some distant part of the system. In cases where it reaches the lungs, it there produces firm spherical patches of livid induration, not unlike in appearance to those observed in the experiment last quoted, 
but differing altogether from them in consistency. In the one case, the patches of congested are produced by the firm coagulation of portions of contaminated blood; in the other, the blood has lost its vital properties together with its power of coagulating, and is already in a state of incipient putrefaction.

In the first instance, the affected portions of lung are at first firm, and an interval is required before they can become softened down; in the other, dissolution commences in the soft, pulpy portions from the first. These general considerations will enable us to trace the progress of disease from a portion, and often a very small portion, of injured cancellous structure of bone to the general system; and to account for the lapse of time which, in the great majority of cases, occurs between the receipt of the injury and the development of its constitutional effects.

When a portion of the cancellous structure of a bone has been injured, the blood in the Haversian canals of the injured part becomes necessarily stagnant between the injury and the next communicating branches. These stagnant portions of blood usually become coagulated and gradually absorbed. If the wound in the bone should suppurate, they at first serve the very important purpose of closing the vessels against the secretions of the part: an office which is subsequently much more permanently performed by the process of adhesive inflammation. It will occasionally happen here, as in the larger veins of the body, that the contents of the injured blood-ressels will become mixed to a greater or less extent with the secretions with which they are in contact. This may arise from some accidental mechanical cause, or from some inherent defect in the coagulating power of the blood.

The admixture of diseased secretions will determine the coagulation of those portions of the blood with which it comes in contact, and thus the coagula will extend farther along the vessels than they would have done, had the blood at first firmly sealed the divided extremities of the veins. Uncontaminated blood will remain for a long time in the living body, either in its solid or fluid state, without undergoing much alteration; but this is not the case when the blood has become mixed with vitiated fluids. Farther changes will then readily take place in it; and we may judge of what takes place in the minute vessels of bone, by that which can be better observed in the larger veins of the body.

There appears no doubt, from evidence derived from direct experiment, that a coagulum formed of vitiated blood may, and often does, in healthy states of the constitution, become gradually and entirely absorbed and eliminated from the system by the intestinal and hepatic secretions; but at other times, the vitiated and perhaps loosely formed coagulum becomes softened down and poured into the adjacent veins. It here determines one of the three following physical results.

1. The dissolved matter of the first formed coagulum mixing with fresh portions of blood may lead to the formation of fresh coagula. These may retain for a time the vitiated fluid; and, adhering at intervals to the sides of the ressels in which they are contained, they may prevent any of the foreign matter from reaching the general circulation. Within a short period, however, the centre of the newly formed coagula will become softened and gradually deprived of its colouring matter. The process of softening will proceed from the 
centre towards the circumference of each portion, until nearly the whole is converted into a white fluid resembling pus. Should there be an opening in the bone, this may escape externally; but otherwise, in. creasing in quantity by the secretions of the surrounding parts, it is forced forward in the course of the circulation to contaminate and mix with fresh portions of blood, which, in their turn, first become coagulated, and then softened down, and converted into the same purulent looking fluid. If an opening be made in a bone in which this process is going on, not only the cancellons structure, but the Haversian canals will appear filled with pus. It occasionally happens under these circumstances, that on making an opening into a bone, a distinct little jet of purulent looking fluid may be seen to escape from one of the canals of the nutritious vessels. In such a case, the cavities of the vessels must of course be accurately closed by firmly adhering coagula in the surrounding parts.

The effects of purulent contamination of the blood usually extend in the course of the circulation.

CA8E. A man, twenty-six years of age, had a blow upon the head which fractured his skull, causing at the same time a scalp wound, which left a portion of the bone denuded. Eighteen days afterwards, he complained of pain in the head, accompanied by nausea. To this succeeded what he termed "soreness of the stomach", which was soon followed by drowsiness and insensibility. He then became paralysed, and a good deal of irregular muscular twitching was observed in different parts of his body. He died a week after the commencement of the above symptoms. Yellow matter was found in the diploë of the parietal bone in the neighbourhood of the fracture, and purulent looking fluid, mixed with flakes of fibrin or lymph, occupied the posterior half only of the longitudinal sinus.

It sometimes, however, happens that the effects of the disease extend in a course opposite to that of the circulation. In these instances, it is probable that the whole mass of the blood has become, to a certain extent, contaminated, those portions which are kept at rest being the most liable to become first coagulated and then softened down. This is not unfrequently observed in cases of infection of the blood after childbirth. The blood in the common iliac vein will become coagulated in consequence of some diseased fluid poured into it from the uterus. This will cause the blood in the femoral and saphena veins to stagnate. Portions of the contaminated and stagnant blood in these, and in the vessels which supply them, will then become coagulated and undergo subsequent changes, involving the coats of the veins and neighbouring parts. Thus the disease will appear to have extended downwards, in a course contrary to that of the circulation. In the same way we must explain the curious fact, that after amputation of one leg or thigh, an abscess will occasionally form in one of the veins of the opposite limb. In such cases, post-mortem examinations sometimes show that the coagulation of the blood has extended from the amputated limb to the vena cava. An obstruction there would of course act equally upon both sides of the body. It is a remarkable fact in these cases, that the coagulum will never extend beyond the opening of the hepatic veins; the double current from the portal system and from the reins opening into the inferior cara being sufficient to carry forward any viscid contents of the vessel. 
2. When the blood has become infected, instead of coagulating, it may separate into its different elements. In this case the fibrin is left unmixed with either the serum or the colouring matter of the blood. This process differs from that of coagulation in this, among other important particulars, namely, that whereas a coagulum formed of all the parts of the blood fills the vessel which contains it, the separation of the blood into its different parts leaves the fibrin alone in the vessel, and allows the more fluid parts to pass on into the general circulation. The consequence of this is that the vessels in which such an action has taken place still remain pervious. The blood can still pass between the fibrinous deposit and the walls of the vein in sufficient quantity to carry on, although imperfectly, the natural circulation of the part; such a condition, unlike that of coagulation of the blood, offers no security against the passage of any diseased secretions along the vessels. This tendency to the separation of the blood into its different parts was strongly marked in the blood which has been detained in the saphena vein in the first case above related. It would appear to be an action which not very unfrequently takes place in the body, although necessarily difficult to illustrate with regard to the smaller veins. I have lately, however, had an opportunity of observing this change in the capillaries of the liver. From each small tube a delicate thread of fibrin could be drawn, leaving some of the fluid parts of the blood in the vessels, which were stained of a darker colour than natural.

A case lately published in the Gazette Médicale de Paris, affords a very good example of this separation of the fibrin from the other elements of blood. The case was that of a soldier, who died in St. Michael's Hospital, having suffered for a long period with symptoms of disease of the chest. Four months before his admission into the hospital, without any apparent cause, and in a single day, the eyelids and the cheeks became considerably swollen. The lower extremities and the forearms became at the same time cdematous. Upon a post-mortem examination, the vessels of the left lung were found to contain fibrinous concretions, having the form of ramified cylinders, which extended through the pulmonary artery into the right ventricle of the heart. These concretions were white, or of a rus-nut colour, solid and resisting. They adhered but very slightly here and there to the lining membrane of the vessels, and were in some parts of the pulmonary artery, and of its larger branches, surrounded by a layer of semi-fluid black blood.

A similar condition has been observed with regard to the vessels of the brain; the longitudinal sinus has been observed to contain a firm yellow fibrinous mass, extending on the one hand in an arborescent form to the vessels of the pia mater, and on the other to the jugular veins.

In such instances, the sudden increase of the symptoms may mark with tolerable certainty the period at which the greater part of the fibrin is deposited, but this would appear to take place gradually in the majority of instances; and it is probable, as in the case of the soldier above mentioned, that it may exist for a long period, during which the circulation may be carried on sufficiently for the purposes of life. Upon a post-mortem inspection, the firm decolorised fibrin may vor. IV. 
at once be distinguished from the blood which has coagulated around it after death.

3. The contaminated blood, instead of coagulating, or of separating into its different elements, may decompose. The whole of the constituents of the blood are then together involved in the changes which take place. The experiments above related shew that the same impediments will not, under such circumstances, be offered to the passage of morbid matter into the circulation as when the blood firmly coagulates in the veins. An interval, however, even in the most strongly marked cases usually elapses between the development of the infecting cause and the manifestation of constitutional symptoms. This is especially the case where diseased secretions enter the circulation through the nutritious vessels of bone. The morbid matter is detained for a certain time, during which the process of decomposition is established. The first infected portions of blood, together with the morbid matters which they contain, then pass on to infect the blood in adjacent vessels. The dissolved and putrifying fibrin from these will proceed further towards the centre of the circulation; in its course it will loosely coagulate fresh portions of blood, and then determine their decomposition. Each fresh portion of blood that is infected will add to the quantity of putrid dissolved fibrin in the vessels, and thus the disease will propagate itself, quite independent of the original source whence the morbid matter was derived. Each portion of blood which is attacked loses its vitality, passes into a state of decomposition, and becomes itself the means of infecting other portions. The contaminated blood may then be found in the vessels in every stage of decomposition, or it may have passed out of the vessels, having stained them during its decomposition of a deep livid colour. Long tracks of deep purple veins will occasionally be found, some being blocked up with the viscid blood in various stages of decomposition, and some having discharged their contents, and being comparatively empty.

CAsE. A man of rather intemperate habits, received a kick from a horse on the right leg, on the 15th of October, 1851. The right tibia and fibula were fractured, and the fracture communicated with a wound in the skin. He was purged, and kept upon low diet. On the 22nd, some redness was observed around the wound, and the cellular tissue in the neighbourhood felt boggy. On the 1st November, he romited after taking food, and on the following days he had several attacks of shivering. On the 4th, his complexion was yellow; he experienced no pain. Five days after this he died.

No union had taken place between the fractured extremities of the tibia, and the parts immediately around the broken portions of the fibula were in a sloughing condition. The superficial and deep veins of the leg and thigh were greatly distended with dark thick blood, but contained no coagula. The iliac veins contained small soft and dark coagula, but were otherwise healthy. In the middle of the right lung were two patches of secondary inflammation, and the right lobe of the liver contained several secondary abscesses, surrounded by firm and dark texture.

The examination of the following case, conducted by Dr. J. W. Ogle, I had the opportunity of witnessing through the kindness of Dr. Wilson, of St. George's Hospital. A married woman, twenty-three years of 
age, miscarried, during the sixth month of her pregnancy, on the 4th of June, 1851. A few days afterwards she was attacked with intense pain in the abdomen, and two or three days later with pain in the calf of the left leg. The pain in the leg was accompanied by some swelling, which afterwards extended up to the thigh. She died on the 11th of July.

A small putrid abscess occupied the course of one of the branches of the left hypogastric vein, at a short distance from the neck of the uterus. The iliac and the femoral veins of the same side were filled with blood in every stage of decomposition. The spermatic vein of the same side was stained of a dark purple colour, but its canal was pervious and contained no coagula.

When the blood in one of the larger veins of the body decomposes, as in the preceding case, the period at which severe constitutional symptoms follow is comparatively short. Analogous affections originating in the minute vessels of bone usually require a longer period for their development. This is illustrated in the following cases.

H. B., aged 19, sustained a fracture of the fibula. Three months after the accident, and three weeks before his death, he had an attack of diffuse cellular inflammation in the leg, which terminated in suppuration of the knee-joint. Upon examining the limb, the tibia in the neighbourhood of the fracture was exposed, and its structure was soft and of a black colour.

J. C., aged 45, had an extensive scalp-wound. Nineteen days afterwards, he experienced a rigor, and shortly became paralysed on one side. A portion of the skull, which was exposed, presented a dark green appearance, and when removed with the trephine, was of a putrid odour. The longitudinal sinus in this case contained contaminated blood, and there were secondary abscesses in the left lung.

In some cases, where the most severe constitutional symptoms have followed injuries of bones, the original lesion has appeared of no very great importance, and the surface of bone exposed has been of very limited extent. In the following instance, the original injury was not regarded with any apprehension, yet it proved speedily fatal after the appearance of the symptoms of secondary inflammation.

E. P., aged 50, had a lacerated wound of the foot, which detached a small portion of the base of the fifth metatarsal bone. This bone was also simply fractured towards its centre. After having for some time progressed without any unfavourable symptoms, pain in the chest, a rapid pulse, depression, with delirium, suddenly made their appearance, and were found, on a post-mortem examination, to have arisen from inflammation of the right pleura, and the formation of secondary abscesses in the right lung. The amount of contaminated blood contained in the injured bone must in such instances be very small; and there can be little doubt that recovery would take place much oftener than it does, did not the disease propagate itself in the blood. When this takes place, it is usually indicated by one of the three classes of physical changes above described. The different natural processes which they illustrate may, however, take place in various degrees in the same case; and the condition of the blood upon post-mortem inspection will, in different parts of the vascular system, present corresponding varieties. 